\title{
The Existence and Uniqueness of Random Solution to Itô Stochastic Integral Equation
}

\author{
Hamdin Ahmed Alafif, Caishi Wang \\ School of Mathematics and Information Science, Northwest Normal University, Lanzhou, China \\ Email: hamdin@126.com
}

Received May 8, 2012; revised June 8, 2012; accepted June 15, 2012

\begin{abstract}
The objective of this paper is to attempt to apply the theoretical techniques of probabilistic functional analysis to answer the question of existence and Uniqueness of a Random Solution to Itô Stochastic Integral Equation. Another type of stochastic integral equation which has been of considerable importance to applied mathematicians and engineers is that involving the Itô or Itô-Doob form of stochastic integrals.
\end{abstract}

Keywords: Itô Integral; Brownian Motion; Probabilistic Functional Analysis; Banach Space

\section{Introduction}

We shall give some historical remarks concerning the development of this type of equation and point out the essential difference between them and other random integral equations.

In $1930 \mathrm{~N}$. Wiener introduced an integral of the form

$$
\int_{a}^{b} g(\tau) \mathrm{d} B(\tau)
$$

where $g(t)$ a deterministic real-valued function and $\{B(\tau), \tau \in[a, b]\}$ is a scalar Brownian motion process.

Author of [1] in 1944 generalized Wiener's integral to include those cases where the integrand is random. That is he obtained an integral of the form

$$
\int_{0}^{t} g(\tau ; w) \mathrm{d} B(\tau), t \in[0,1]
$$

Which is referred to as the Itô stochastic integral or simply the stochastic integral. Since that time many scientists have contributed to the general development of this type of stochastic integral. For example see [210].

In 1946 Author of [5] formulated a stochastic integral equation of the form

$$
\begin{aligned}
x(t ; w)= & C+\int_{0}^{t} f(\tau, x(\tau ; w)) \mathrm{d} \tau \\
& +\int_{0}^{t} g(\tau, x(\tau ; w)) \mathrm{d} B(\tau)
\end{aligned}
$$

where $t \in[0,1], \quad\{B(t) ; t \in[0,1]\}$ is a scalar Brownian motion process, and $C$ is a constant Restrictions are usually placed on the functions $f$ and $g$ so that the first integral is interpreted as the usual Lebesgue integral of the sample functions which can then be related to the sample integral of the process $\{f(t, x(t ; w), t \in[0,1]\}$ and the second integral is an Itô stochastic integral.

The principal feature which distinguishes the type of equation studied from an equation of the Itô type is the fact that in the former case each of the integrals involved is interpreted as a Lebesgue integral for almost all $w \in \Omega$. That is, almost all sample functions are Lebesgue integrable. Since in the Itô stochastic integral the limit is taken in the mean-square or in the probability sense, the theory of such integrals has been developed as self-contained and self-consistent.

One of the main purposes of subsequent work in connection with the Itô stochastic integral equation has been to construct Markov processes such that their transition probabilities satisfy given Kolmogorov equations and to investigate the continuity of the processes, among other properties of the sample function.

The method of successive approximation was used by Itô and Doob to show the existence and uniqueness of a random solution to Equation (1.0).

\section{Preliminaries}

Let $\{B(t) ; t \in[a, b]\}$ be a scalar Brownian motion process. In this section we shall be concerned with the integral

$$
\int_{a}^{b} \phi(t ; w) \mathrm{d} B(t) a<b
$$

for a fairly general class of functions $\phi$. This integral will be called the Itô stochastic integral as we mentioned previously. As is well known, almost all the sample functions of the Brownian motion process are of un- 
bounded variation and hence the integral (1.1) cannot be defined as an ordinary Stieltjes integral.

First we shall define the integral (1.1) for the class of step functions. That is, functions $\phi$ of the form

$$
\phi(t ; w)=\left\{\begin{array}{cc}
0 & t<a \\
\phi_{i}(w), & t_{i} \leq t \leq t_{i+1} \\
0 & t>b
\end{array}\right.
$$

where $a=t_{0}<t_{1}<t_{2}<\cdots<t_{n-1}<t_{n}=b, \phi_{i}(w)$ are measurable with respect to the $\sigma$-algebra $A_{t_{i}}$, and $E\left\{\left|\phi_{i}(w)\right|^{2}\right\}<\infty$ for such functions we define the Itô integral by

$$
\int_{a}^{b} \phi(t ; w) \mathrm{d} B(t)=\sum_{i=0}^{n-1} \phi_{i}(w)\left(B\left(t_{i+1}\right)-B\left(t_{i}\right)\right)
$$

Now suppose that $\phi(t ; w)$ is any function satisfying the following conditions.

1) $\phi(t ; w)$ is a product-measurable function from $[a, b] \times \Omega \rightarrow \mathbb{R}_{+}$, assuming the usual Lebesgue measure on $\mathbb{R}_{+}$.

2) For each, $t \in[a, b], \phi(t ; w)$ is measurable with respect to $\sigma$-algebra $A_{t}$, where $A_{t}$ is the smallest $\sigma$-algebra on $\Omega$, such that $B(s), s \leq t$ is measurable.

3) $\int_{-\infty}^{\infty} E|\phi(t ; w)|^{2} \mathrm{~d} t<\infty$

In view of Equation (1.2) it is evident that the class of step functions satisfy conditions 1)-3).

For the function $\phi(t ; w)$ satisfying conditions 1)-3) we shall define their norm as follows:

$$
\|\phi(t ; w)\|=\left\{\int_{a}^{b} E[|\phi(t ; w)|]^{2} \mathrm{~d} t\right\}^{\frac{1}{2}}
$$

For this case author of [2] has shown the following

1) $\phi(t ; w)$ can be approximated in the mean-square sense by a sequence of step functions $\left\{\phi_{n}(t ; w)\right\}$. That is

$$
\left\|\phi(t ; w)-\phi_{n}(t ; w)\right\| \rightarrow 0 \text { as } n \rightarrow \infty
$$

2) The sequence of integrals

$$
\int_{a}^{b} \phi_{n}(t ; w) \mathrm{d} B(t)
$$

Possesses a mean-square limit. That is there exists a $\theta(w)$ such that

$$
E\left\{\theta(w)-\int_{a}^{b} \phi_{n}(t ; w) \mathrm{d} B(t)\right\}^{2} \rightarrow 0
$$

as $n \rightarrow \infty$

Now we shall define the integral (1.1) for a class of functions $\{\phi(t ; w)\}$ satisfying conditions 1)-3) by

$$
\int_{a}^{b} \phi(t ; w) \mathrm{d} B(t)=\theta(w)
$$

As with the ordinary integrals, we shall define

$$
\int_{-\infty}^{\infty} \phi(t ; w) \mathrm{d} B(t)=\lim _{a \rightarrow-\infty, b \rightarrow+\infty} \int_{a}^{b} \phi(t ; w) \mathrm{d} B(t)
$$

Definition 1.1 Let $G \in L$, where $L$ denote the collection of Lebesgue measurable subsets of $\mathbb{R}_{+}$. Define a function $\chi_{G}$ from $\mathbb{R}_{+} \times \Omega \rightarrow\{0,1\}$ by

$$
\chi_{G}(\tau ; w)=\left\{\begin{array}{cc}
1 & \text { if }(\tau ; w) \in G \times \Omega \\
0 & \text { otherwise }
\end{array}\right.
$$

Lemma 1.1 The function $\varphi \chi_{G}: \mathbb{R}_{+} \times \Omega \rightarrow \mathbb{R}_{+}$defined by

$$
\left(\varphi \chi_{G}\right)(\tau ; w)=\varphi(\tau ; w) \chi_{G}(\tau ; w)
$$

where $\phi$ satisfies conditions 1)-3), and $\chi_{G}$ is as defined earlier, also satisfies conditions 1)-3).

Proof. The proof is a straightforward result of the definition of $X_{G}$ and the fact that $\phi$ satisfies conditions 1)-3).

We are now in a position to define exactly what is meant by the expression

$$
\int_{G} \phi(\tau ; w) \mathrm{d} B(\tau)
$$

Definition 1.2 We define $\int_{G} \phi(\tau ; w) \mathrm{d} B(\tau)$ for $G$ a Lebesgue-measurable subset of $\mathbb{R}_{+}$by

$$
\int_{G} \varphi(\tau ; w) \mathrm{d} B(\tau)=\int_{-\infty}^{\infty}\left(\varphi \chi_{G}\right)(\tau ; w) \mathrm{d} B(\tau)
$$

Note that lemma 1.4 guarantees the expression on the right exists and is well defined

Definition 1.3 We shall denote by

$C^{*}\left([a, b], L_{2}(\Omega, \mathcal{F}, P)\right)$ the space of all continuous functions from $[a, b]$ into $L_{2}(\Omega, \mathcal{F}, P)$. We shall define the norm of $C^{*}\left([a, b], L_{2}(\Omega, \mathcal{F}, P)\right)$ by

$$
\sup _{a \leq t \leq b}\left\{\int_{\Omega}|x(t ; w)|^{2} \mathrm{~d} P(w)\right\}^{\frac{1}{2}}
$$

\section{Lemma 1.2}

$$
E[\theta]=E\left[\int_{-\infty}^{\infty} \phi(t ; w) \mathrm{d} B(t)\right]=0
$$

\section{Lemma 1.3}

$$
E\left[|\theta|^{2}\right]=\int_{-\infty}^{\infty} E\left[|\phi(t ; w)|^{2}\right] \mathrm{d} t
$$

Lemma 1.4 If we define a distance between two functions $\phi_{1}$ and $\phi_{2}$ each satisfying conditions 1)-3) by

$$
\left\|\phi_{1}-\phi_{2}\right\|=\left\{\int_{-\infty}^{\infty} E\left|\phi_{1}(t ; w)-\phi_{2}(t ; w)\right|^{2} \mathrm{~d} t\right\}^{\frac{1}{2}}
$$

and the distance between $\theta_{1}=\int_{-\infty}^{\infty} \phi_{1}(t ; w) \mathrm{d} B(t)$ and $\theta_{2}=\int_{-\infty}^{\infty} \phi_{2}(t ; w) \mathrm{d} B(t)$ by 


$$
\left\|\theta_{1}-\theta_{2}\right\|=\left\{E\left[\left|\theta_{1}-\theta_{2}\right|^{2}\right]\right\}^{\frac{1}{2}}
$$

Then $\left\|\theta_{1}-\theta_{2}\right\|=\left\|\phi_{1}-\phi_{2}\right\|$.

For the proof of the Lemmas see [2].

Lemma 1.5 Let $x(t ; w)=\int_{a}^{t} \phi(\tau ; w) \mathrm{d} B(\tau), \quad t \in[a, b]$

Then $x(t ; w) \in C^{*}\left([a, b], L_{2}(\Omega, \mathcal{F}, P)\right)$

For the proof see [4].

\section{On an Itô Stochastic Integral Equation}

In this section we shall investigate a stochastic integral equation of the type

$$
\begin{aligned}
x(t ; w)= & \int_{0}^{t} k(t, \tau ; w) f(\tau, x(\tau ; w)) \mathrm{d} \tau \\
& +\int_{0}^{t} \phi(\tau ; w) \mathrm{d} B(\tau) \quad t \geq 0
\end{aligned}
$$

where $x(t ; w)$ is the unknown random process defined for $t \in \mathbb{R}_{+}$and $w \in \Omega$.

We shall place the following restrictions on the random functions which constitute the stochastic integral Equation (2.1).

$\left.1^{\prime}\right) \quad k(t, \tau ; w)$ is an element of $L_{\infty}(\Omega, \mathcal{F}, P)$ and $k(t, \tau ; w): \Delta \rightarrow L_{\infty}(\Omega, \mathcal{F}, P)$ is continuous where $\Delta=\{(t, \tau): 0 \leq \tau \leq t<\infty\}$.

2') $x(t ; w) \rightarrow f(t, x(t ; w))$ is an operator on the set $S$ with values in the Banach space $B$ satisfying

$$
\begin{aligned}
& \|f(t, x(t ; w))-f(t, y(t ; w))\|_{B} \\
& \leq \lambda\|x(t ; w)-y(t ; w)\|_{D}
\end{aligned}
$$

for $x(t ; w), y(t ; w) \in S$.

3 ) Conditions 1)-3) of section 1 hold.

Thus with the given assumptions the first integral of (2.1) can be interpreted as a Lebesgue integral and the second as an Itô stochastic integral.

We shall now proceed to state and prove a theorem concerning the behavior of the Itô integral. More precisely, if we show that the Itô integral is an element of the space $C_{C}\left(\mathbb{R}_{+}, L_{2}(\Omega, \mathcal{F}, P)\right)$, we can apply the theory of admissibility to Equation (2.1) to show the existence of a random solution. By a random solution to Equation (2.1) we mean a random function $x(t ; w)$ from $\mathbb{R}_{+}$into $L_{2}(\Omega, \mathcal{F}, P)$ such that for each $t \in \mathbb{R}_{+}$, $x(t ; w)$ satisfies the integral equation $P$-a.e. showing that the Itô integral is an element of $C_{C}\left(\mathbb{R}_{+}, L_{2}(\Omega, \mathcal{F}, P)\right)$ will make feasible the assumption that we wish to make that the integral is an element of $D$, a Banach space contained in the topological space mentioned

For convenient we shall denote the Itô integral by

$$
h(t ; w)=\int_{0}^{t} \phi(\tau ; w) \mathrm{d} B(\tau), \quad t \geq 0
$$

\section{Theorem 2.1 For}

$$
t \in \mathbb{R}_{+}, h(t ; w) \in C_{C}\left(\mathbb{R}_{+}, L_{2}(\Omega, \mathcal{F}, P)\right)
$$

Proof Fix $t \in \mathbb{R}_{+}$Then

$$
h(t ; w)=\int_{0}^{t} \phi(\tau ; w) \mathrm{d} B(\tau)=\int_{-\infty}^{\infty}\left(\phi X_{[0, t]}\right)(\tau ; w) \mathrm{d} B(\tau)
$$

Thus

$$
\begin{aligned}
E|h(t ; w)|^{2} & =E\left|\int_{-\infty}^{\infty}\left(\phi x_{[0, t]}\right)(\tau ; w) \mathrm{d} B(\tau)\right|^{2} \\
& =\int_{-\infty}^{\infty} E\left|\left(\phi x_{[0, t]}\right)(\tau ; w)\right|^{2} \mathrm{~d} \tau
\end{aligned}
$$

by lemma 1.3 .

Hence $E|h(t ; w)|^{2}<\infty$.

Therefore for fixed $t, h(t ; w) \in L_{2}(\Omega, \mathcal{F}, P)$. Now let $t_{n} \rightarrow t$ in $\mathbb{R}_{+}$. To show that $h_{n}(t ; w) \rightarrow h(t ; w)$ in $L_{2}(\Omega, \mathcal{F}, P)$, it is sufficient to show that

$$
\left\|\phi x_{\left[0, t_{n}\right]}-\phi x_{[0, t]}\right\|
$$

can be made arbitrarily small. That is, we must show that

$$
\int_{-\infty}^{\infty} E\left|\left(\phi x_{\left[0, t_{n}\right]}\right)(\tau ; w)-\left(\phi x_{[0, t]}\right)(\tau ; w)\right|^{2} \mathrm{~d} t
$$

Can be made arbitrarily small. Choose $\varepsilon>0$. Consider the nonnegative function $q(\tau ; w)=E|\phi(\tau ; w)|^{2}$. By condition 3) $q(\tau ; w)$ is integrable over $\mathbb{R}_{+}$. Hence there exists a $\delta>0$ such that for every set of Lebesgue measure less than $\delta, \int_{G} q(\tau ; w) \mathrm{d} \tau<\infty$. Thus

$$
\begin{aligned}
& \int_{-\infty}^{\infty} E\left|\left(\phi x_{\left[0, t_{n}\right]}\right)(\tau ; w)-\left(\phi x_{[0, t]}\right)(\tau ; w)\right|^{2} \mathrm{~d} t \\
& =\int_{t}^{t_{n}} E\left|\left(\phi x_{\left[0, t_{n}\right]}\right)(\tau ; w)-\left(\phi x_{[0, t]}\right)(\tau ; w)\right|^{2} \mathrm{~d} \tau \\
& =\int_{t}^{t_{n}} E|\phi(\tau ; w)|^{2} \mathrm{~d} \tau=\int_{t}^{t_{n}} q(\tau ; w) \mathrm{d} \tau
\end{aligned}
$$

Since for $n>N_{\delta}$ and $\left|t_{n}-t\right|<\delta$ and since the Lebesgue measure of the interval $\left(t, t_{n}\right)$ is its length, we conclude that the Lebesgue measure of $\left(t, t_{n}\right)$ is less than $\delta$.

Hence

$$
\int_{t}^{t_{n}} q(\tau ; w) \mathrm{d} \tau<\varepsilon
$$

Implying that $t \rightarrow h(t ; w)$ is continuous from $\mathbb{R}_{+}$ into $L_{2}(\Omega, \mathcal{F}, P)$ and the proof is complete.

Since we have shown that $h(t ; w) \in C_{C}\left(\mathbb{R}_{+}, L_{2}(\Omega, \mathcal{F}, P)\right)$, we can conclude that the stochastic integral Equation (2.1) possesses a unique random solution

\section{On Itô-Doob-Type Stochastic Integral Equations}

In this section we shall study the existence and unique- 
ness of a random solution to a stochastic integral equation of the form

$$
x(t ; w)=\int_{0}^{t} f(\tau, x(\tau ; w)) \mathrm{d} \tau+\int_{0}^{t} \phi(\tau, x(\tau ; w)) \mathrm{d} B(\tau)
$$

where $t \in[0,1]$. As before, the first integral is a Lebesgue integral, while the second is an Itô-type stochastic integral defined with respect to a scalar Brownian motion process $\{B(t), t \in[0,1]\}$.

Recall that

$C^{*}\left([0,1], L_{2}(\Omega, \mathcal{F}, P)\right) \subset C_{C}\left(\mathbb{R}_{+}, L_{2}(\Omega, \mathcal{F}, P)\right)$, We shall define the operators $W_{1}$ and $W_{2}$ from

$C^{*}\left([0,1], L_{2}(\Omega, \mathcal{F}, P)\right)$ into $C^{*}\left([0,1], L_{2}(\Omega, \mathcal{F}, P)\right)$ by

$$
\left(w_{1} x\right)(t ; w)=\int_{0}^{t} x(\tau ; w) \mathrm{d} \tau
$$

and

$$
\left(w_{2} x\right)(t ; w)=\int_{0}^{t} x(\tau ; w) \mathrm{d} B(\tau)
$$

Note that in view of lemma 1.5 $x(t ; w) \in C^{*}\left([0,1], L_{2}(\Omega, \mathcal{F}, P)\right)$. Its clear that $W_{1}$ and $W_{2}$ are linear operators.

Theorem 3.1 The operators $W_{1}$ and $W_{2}$ defined by (3.2) and (3.3) respectively, are continuous operators from $C^{*}\left([0,1], L_{2}(\Omega, \mathcal{F}, P)\right)$ into $C^{*}\left([0,1], L_{2}(\Omega, \mathcal{F}, P)\right)$.

Lemma 3.1 Let $T$ be a continuous operator from $C_{C}\left(\mathbb{R}_{+}, L_{2}(\Omega, \mathcal{F}, P)\right)$ into itself. If $B$ and $D$ are Banach spaces stronger than $C_{C}$ and the pair $(B, D)$ is admissible with respect to $T$. Then $T$ is a continuous operator from $B$ to $D$.

Proof of theorem 3.1 The fact that $W_{1}$ is a continuous operator from $C^{*}\left([0,1], L_{2}(\Omega, \mathcal{F}, P)\right)$ into

$C^{*}\left([0,1], L_{2}(\Omega, \mathcal{F}, P)\right)$ follows from lemma 3.1. From (3.3) we have

$$
\begin{aligned}
\left\|\left(w_{2} x\right)(t ; w)\right\|_{L_{2}(\Omega, \mathcal{F}, P)}^{2} & =\int_{\Omega} \mathrm{d} P(w)\left\{\int_{0}^{t} x(\tau ; w) \mathrm{d} B(\tau)\right\}^{2} \\
& =\int_{0}^{t} \mathrm{~d} \tau \int_{\Omega} x^{2}(\tau ; w) \mathrm{d} P(w)
\end{aligned}
$$

Furthermore

$$
\begin{aligned}
\left\|\left(w_{2} x\right)(t ; w)\right\|_{L_{2}(\Omega, \mathcal{F}, P)}^{2} & \leq \int_{0}^{t} \mathrm{~d} \tau \sup _{0 \leq \tau \leq t}\|x(\tau ; w)\|_{L_{2}(\Omega, \mathcal{F}, P)}^{2} \\
& =\int_{0}^{t}\left\{\sup _{0 \leq \tau \leq t}\|x(\tau ; w)\|_{L_{2}(\Omega, \mathcal{F}, P)}\right\} \mathrm{d} \tau \\
& =\|x(t ; w)\|^{2} \int_{0}^{t} \mathrm{~d} \tau \leq\|x(t ; w)\|^{2}
\end{aligned}
$$

Therefore

$$
\left\|\left(w_{2} x\right)(t ; w)\right\| \leq\|x(t ; w)\|
$$

Thus $W_{1}$ and $W_{2}$ are continuous operators from $C^{*}\left([0,1], L_{2}(\Omega, \mathcal{F}, P)\right)$ into $C^{*}\left([0,1], L_{2}(\Omega, \mathcal{F}, P)\right)$.

\section{An Existence Theorem}

We shall assume that lemma 3.1 holds with respect to the operators $W_{1}$ and $W_{2}$. Therefore there exist positive constants $K_{1}$ and $K_{2}$ less than one such that $\left\|\left(w_{1} x\right)(t ; w)\right\|_{D} \leq k_{1}\|x(t ; w)\|_{B}$ and $\left\|\left(w_{2} x\right)(t ; w)\right\|_{D} \leq k_{2}\|x(t ; w)\|_{B}$

The following theorem gives sufficient conditions for the existence of a unique random solution, a second order stochastic process, to the Itô-Doob stochastic integral Equation (3.1).

Theorem 3.2 Consider the stochastic integral equation (3.1) under the following condition:

1) $B$ and $D$ are Banach spaces in

$C^{*}\left([0,1], L_{2}(\Omega, \mathcal{F}, P)\right)$ which are stronger than $C^{*}\left([0,1], L_{2}(\Omega, \mathcal{F}, P)\right)$ such that $(B, D)$ is admissible with respect to the operators $W_{1}$ and $W_{2}$

2) a) $x(t ; w) \rightarrow f(t, x(t ; w))$ is an operator on

$$
S=\left\{x(t ; w): x(t ; w) \in D \text { and }\|x(t ; w)\|_{D} \leq \rho\right\}
$$

With values in $B$ satisfying

$\|f(t, x(t ; w))-f(t, y(t ; w))\|_{B} \leq \lambda_{1}\|x(t ; w)-y(t ; w)\|_{D}$

b) $x(t ; w) \rightarrow \phi(t, x(t ; w))$ is an operator on $S$ into $B$ satisfying

$$
\|\phi(t, x(t ; w))-\phi(t, y(t ; w))\|_{B} \leq \lambda_{2}\|x(t ; w)-y(t ; w)\|_{D}
$$

where $\lambda_{1}$ and $\lambda_{2}$ are constants. Then there exists a unique random solution to Equation (3.1) provided that $k_{1} \lambda_{1}+k_{2} \lambda_{2}<1$. And

$$
\|f(t, 0)\|_{B}+\|\phi(t, 0)\|_{B} \leq \rho\left(1-\lambda_{1} k_{1}-\lambda_{2} k_{2}\right)
$$

Proof. Define an operator $U$ from the set $S$ into $D$ as follows

$$
\begin{aligned}
(U x)(t ; w)= & \int_{0}^{t} f(\tau, x(\tau ; w)) \mathrm{d} \tau \\
& +\int_{0}^{t} \phi(\tau, x(\tau ; w)) \mathrm{d} B(\tau)
\end{aligned}
$$

We need to show that $U$ is a contraction operator on $S$ and that $U S \subset S$.

Let $x(t ; w), y(t ; w) \in S$.

Then $(U x)(t ; w)-(U y)(t ; w) \in D$ because $D$ is a Banach space. Further, we have

$$
\begin{aligned}
& \|(U x)(t ; w)-(U y)(t ; w)\|_{D} \\
& \leq\left\|\int_{0}^{t}[f(\tau, x(\tau ; w))-f(\tau, y(\tau ; w))] \mathrm{d} \tau\right\|_{D} \\
& \quad+\left\|\int_{0}^{t}[\phi(\tau, x(\tau ; w))-\phi(\tau, y(\tau ; w))] \mathrm{d} B(\tau)\right\|_{D} \\
& \leq k_{1}\|f(t, x(t ; w))-f(t, y(t ; w))\|_{B} \\
& \quad+k_{2}\|\phi(t, x(t ; w))-\phi(t, y(t ; w))\|_{B} \\
& \leq\left(\lambda_{1} k_{1}+\lambda_{2} k_{2}\right)\|x(t ; w)-y(t ; w)\|_{D}<\|x(t ; w)-y(t ; w)\|
\end{aligned}
$$


Thus $U$ is a contraction operator.

For any element in $S$ we have

$$
\begin{aligned}
& \|(U x)(t ; w)\|_{D} \\
& \leq\left\|\int_{0}^{t} f(\tau, x(\tau ; w)) \mathrm{d} \tau\right\|_{D}+\left\|\int_{0}^{t} \phi(\tau, x(\tau ; w)) \mathrm{d} B(\tau)\right\|_{D} \\
& \leq k_{1}\|f(t, x(t ; w))\|_{B}+k_{2}\|\phi(t, x(t ; w))\|_{B} \\
& \leq \lambda_{1} k_{1}\|x(t ; w)\|_{D}+\lambda_{2} k_{2}\|x(t ; w)\|_{D} \\
& +k_{1}\|f(t, 0)\|_{B}+k_{2}\|\phi(t, 0)\|_{B}
\end{aligned}
$$

Since $x(t ; w) \in S$ it follows that

$$
\begin{aligned}
& \|(U x)(t ; w)\|_{D} \\
& \leq \rho\left(\lambda_{1} k_{1}+\lambda_{2} k_{2}\right)+\|f(t, 0)\|_{B}+\|\phi(t, 0)\|_{B} \leq \rho
\end{aligned}
$$

from the assumptions in the theorem.

Thus the existence and uniqueness of a random solution to Equation (3.1) follow from the Banach fixed-point theorem.

Theorem 3.4 (S. Banach's fixed-point principle) ([11]).

If $T$ is a contraction operator on a complete metric space $H$. then there exists a unique point $x^{*} \in H$ for which $T\left(x^{*}\right)=x^{*}$.

\section{Conclusion}

We investigated the existence and uniqueness of Itô stochastic integral equation by applying the theoretical techniques of probabilistic functional analysis. In fact author of [12] refers to probabilistic functional analysis as being concerned with the applications and extensions of the methods of functional analysis to the study of the various concepts, processes, and structures which arise in the theory of probability and its applications. Finally to develop and unify the theory of stochastic or random equations see [13-15].

\section{REFERENCES}

[1] K. Ito, "Stochastic Integral," Proceedings of the Imperial Academy, Vol. 20, No. 8, 1944, pp. 519-524. doi:10.3792/pia/1195572786

[2] J. L. Doob, “Stochastic Processes," Wiley, New York, 1953, pp. 426-432.

[3] Y. Dynkin, "Markov Processes,” Academic Press, New York, 1964, pp. 9-13.

[4] A. Jazwinski, "Stochastic Processes and Filtering Theory. Mathematics in Science and Engineering," Vol. 64, Academic Press, New York, 1970, pp. 97-105

[5] K. Ito, “On a Stochastic Integral Equation,” Proceedings of the Japan Academy, Vol. 22, No. 2, 1946, pp. 32-35. doi:10.3792/pja/1195572371

[6] H. P. Mckean, "Stochastic Integrals,” Academic Press, New York, 1969, pp. 21-25.

[7] T. L. Satty, "Modern Nonlinear Equations," McGrowHill, New York, 1967, pp. 216-226.

[8] L. Gikhmann and A. V. Skorokhod, "Introduction to the Theory of Random Process-Saunders,” Philadehphia, Pennsylvania, 1969, pp. 378-391.

[9] R. L. Stratonovich, "A New Representation for Stochastic Integrals and Equations,” Journal of SLAM Control, Vol. 4, 1966, pp. 362-371.

[10] E. Wong and M. Zakai, "On the Relation between Ordinary and Stochastic Differential Equations," International Journal of Engineering Science, Vol. 3, No. 2, 1965, pp. 213-229. doi:10.1016/0020-7225(65)90045-5

[11] I. P. Natanson, "Theory of Functions of a Real Variable," Vol. II, Ungar, New York, 2010.

[12] A. T. Bharucha-Reid, "On the Theory of Random Equations," Proceedings of Symposia in Applied Mathematics, Vol. 16, 1964, pp. 40-69.

[13] G. Adomain, "Random Operator Equations in Mathematical Physics,” Journal of Mathematical Physics, Vol. 11, No. 3, 1970, pp. 1069-1074. doi:10.1063/1.1665198

[14] G. Adomain, "Linear Random Operator Equations in Mathematical Physics III," Journal of Mathematical Physics, Vol. 12, No. 9, 1971, pp. 1944-1948. doi:10.1063/1.1665827

[15] G. Adomain, “Theory of Random Systems,” Transactions of the fourth Prague Conference on Information Theory, Statistical Decision Functions, Random Processes, Prague, 31 August-11 September 1965, pp. 205-222. 